\title{
Differential Measurement and Model Calculations of Cosmic Ray Latitudinal Gradient With Respect to the Heliospheric Current Sheet
}

\author{
S. P. Christon, A. C. Cummings, and E. C. Stone \\ California Institute of Technology, Pasadena
}

K. W. Behannon and L. F. Burlaga

Goddard Space Flight Center, Greenbelt, Maryland

J. R. JoKIPII AND J. KoTA ${ }^{1}$

University of Arizona, Tucson

\begin{abstract}
Simultaneous magnetic field and charged particle measurements from the Voyager spacecraft with heliographic latitude separations of $>10^{\circ}$ are used to investigate the distribution of $\sim 1-\mathrm{GeV}$ galactic cosmic ray protons with respect to the heliospheric current sheet in the outer solar system. By comparing the ratio of cosmic ray flux at Voyager 1 to that at Voyager 2 during periods of relatively quiet interplanetary conditions when the spacecraft are either both north or both south of the heliospheric current sheet, we derive an average latitude component of the gradient of the cosmic ray flux on opposite sides of the current sheet under restricted interplanetary conditions of $-0.22 \pm 0.03 \% / \mathrm{deg}$, equivalent to a decrease of $\sim 1 \% / \mathrm{AU}$ away from the current sheet at $\sim 12 \mathrm{AU}$. Our results for these limited periods are in qualitative agreement with propagation models incorporating particle drifts.
\end{abstract}

\section{INTRODUCTION}

The latitudinal variation in the intensity of galactic cosmic rays with respect to the heliospheric current sheet is an indicator of the relative importance of particle drifts and diffusion in heliospheric propagation. Symmetries in the interplanetary magnetic field due to the sun's rotation, i.e., wrapped up spiral field lines at the equator in contrast to nearly radial field lines at either pole, should result in a latitudinal organization of the flux about the heliograhic equator [Fisk, 1976; Newkirk and Fisk, 1985]. Transport models including only the effects of diffusion, convection, and adiabatic deceleration can produce latitudinal variations of cosmic ray flux for selected choices of the model parameters [see Newkirk and Fisk, 1985, and references therein], but in these two-dimensional models, intensity minima are symmetrically displaced with respect to the heliographic equator, and the effects of azimuthal variations in the solar wind velocity have not been evaluated. In contrast, if particle drifts are important in cosmic ray transport, minima in the cosmic ray flux should be symmetrically placed with respect to the heliospheric current sheet, as has recently been demonstrated in a three-dimensional model of cosmic ray transport including particle drifts [Kota and Jokipii, 1983] and reported in various investigations below.

The geometry of the wavy current sheet is an important factor in propagation models including particle drifts. Examples of the spatial distribution of $1.6-\mathrm{GeV}$ cosmic ray protons at $1 \mathrm{AU}$ and $5 \mathrm{AU}$ predicted by the numerical model of Kota and Jokipii [1983] for the present half of the 22-year solar magnetic activity cycle are displayed in Figure 1. Parameters

\footnotetext{
${ }^{1}$ Permanently at Central Research Institute for Physics, Budapest, Hungary.

Copyright 1986 by the American Geophysical Union.

Paper number 5A8802.

0148-0227/86/005A-8802\$05.00
}

producing differences between the examples are listed in the figure caption. Effects of the wavy current sheet are evident in the isointensity curves at all latitudes and longitudes, although there are significant differences in detail due mainly to the relative importance of drift and diffusion for the two diffusion coefficients used. The calculated intensities depend on a balance among (1) diffusion from the heliospheric boundary, (2) outward convection with the solar wind, (3) sunward drift along the current sheet in this solar epoch, and (4) subsequent diffusion and drifting away from the current sheet. In addition to different tilt angles, the diffusion coefficient $K_{0}$ used in Figures $1 a$ and $1 b$ is a factor of 3 smaller than that used in Figures $1 c$ and $1 d$, with the same $400-\mathrm{km} / \mathrm{s}$ solar wind velocity in both examples. The two values of $K_{0}$ used represent approximate upper and lower limits for $1-$ to $2-\mathrm{GeV}$ protons at 1 AU. As a result of the difference in $K_{0}$, diffusion from the polar regions is much more important in Figures $1 c$ and $1 d$, while the effects of drift and diffusion away from the current sheet are more evident in Figures $1 a$ and $1 b$. There is no single parameter, such as heliographic latitude 9 , or the angular distance from the current sheet $\lambda_{D}$ which will completely organize the complex gradients.

Newkirk and Lockwood [1981], Newkirk and Fisk [1985], and Newkirk et al. [this issue] used heliomagnetic latitude $\lambda_{m g}$ and angular distance from the current sheet $\lambda_{D}$ to organize their observations. In their studies, ground-based synoptic observations of the $K$ corona are used to determine the shape of the current sheet at the corona. This synoptic coronal information was then used in combination with solar wind velocity data to infer the position of the current sheet in latitude and longitude at $1 \mathrm{AU}$ (for details, see Appendix $\mathrm{C}$ of Newkirk and Fisk [1985]). They found a $2-3 \%$ decrease in the average flux of $5-\mathrm{GeV}$ galactic cosmic rays measured at $1 \mathrm{AU}$ for $\$ 45^{\circ}$ displacements from the inferred position of the heliospheric current sheet, giving an average latitudinal gradient with respect to the current sheet $G_{\lambda_{m g}}$ of -0.04 to $-0.07 \% / \mathrm{deg}$. Sub- 

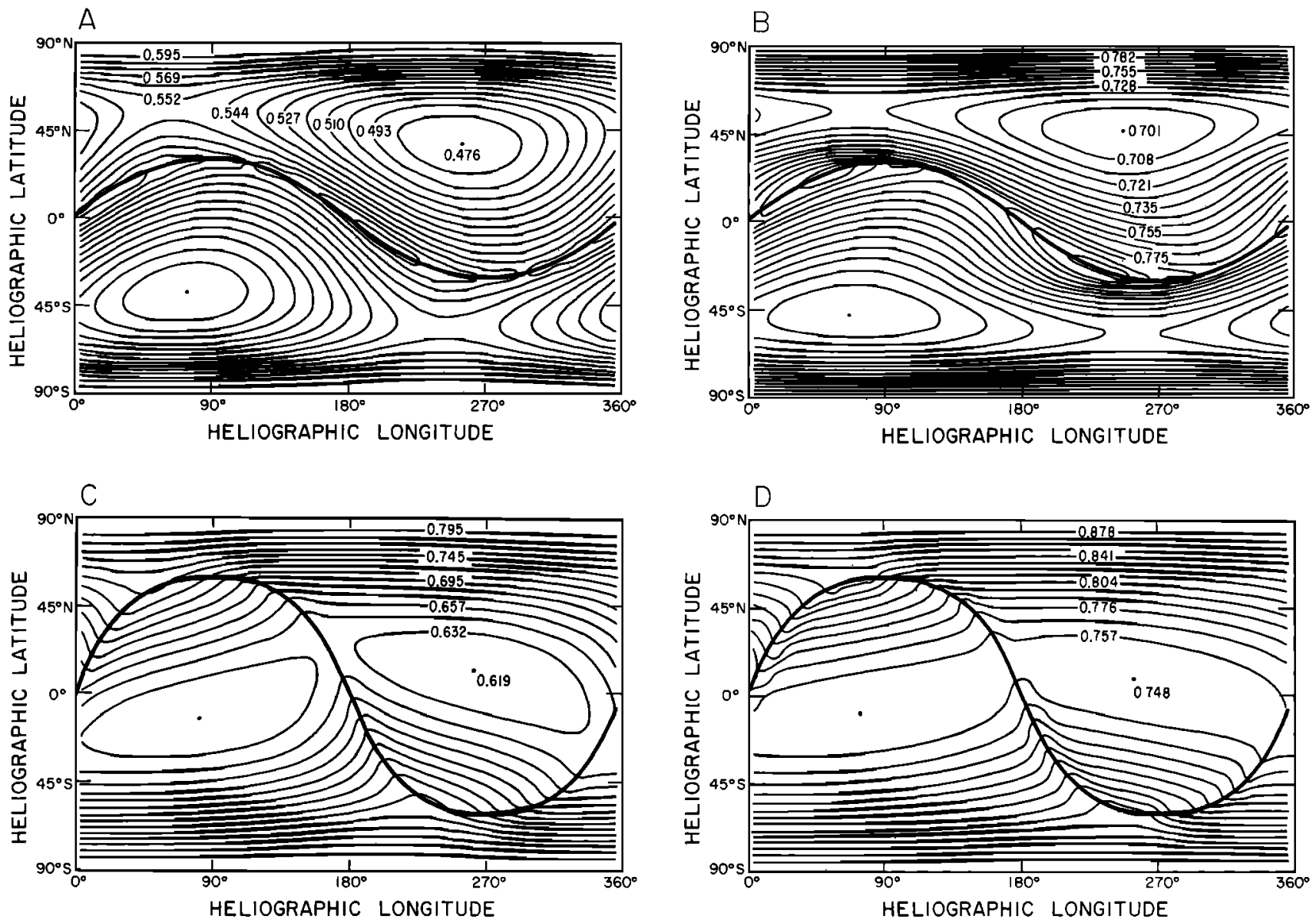

Fig. 1. Model calculations of intensity contours for $1.6-\mathrm{GeV}$ protons near $1 \mathrm{AU}$ and $5 \mathrm{AU}$ for current sheet tilts of $30^{\circ}$ (Figures $1 a$ and $1 b$ ) and $60^{\circ}$ (Figures $1 c$ and $1 d$ ) during the present 11-year portion of the solar cycle. Normalization is made to the interstellar intensity, and the heavy line indicates the position of the current sheet. Other relevant model parameters are (Figures $1 a$ and $1 b) K_{0}=5 \times 10^{21} \mathrm{~cm}^{2} \mathrm{~s}^{-1}$ and $R_{b}=10 \mathrm{AU}$ and (Figures $1 \mathrm{c}$ and $1 d$ ) $K_{0}=1.5 \times 10^{22} \mathrm{~cm}^{2}$ $\mathrm{s}^{-1}$ and $R_{b}=15$ AU. These and other model parameters are defined by Kota and Jokipii [1983].

sequently, Newkirk et al. [this issue] found latitude gradients at $1 \mathrm{AU}$ corresponding to $G_{\lambda_{m g}} \sim-0.4 \% / \mathrm{deg}$ and $-0.03 \% / \mathrm{deg}(\sim-23 \% / \mathrm{AU}$ and $-2 \% / \mathrm{AU}$, respectively) for cosmic rays with kinetic energies of $>100 \mathrm{MeV}$ and $\sim 13$ $\mathrm{GeV}$, respectively. These results are shown by Jokipii and Kota [this issue] to be in reasonably good agreement with models in which drift plays an important role.

Badruddin et al. [1985], using a superposed epoch analysis of neutron monitor measurements of the flux of $\mathrm{GeV}$ energy cosmic rays in combination with observations of the current sheet at $1 \mathrm{AU}$ (i.e., interplanetary magnetic field sector boundaries), demonstrate that cosmic ray intensity generally decreases with increasing time from current sheet encounters and interpret this as indicative of a latitudinal variation, although they do not quantify their results. These observations by Badruddin et al. are consistent with the results of Duggal et al. [1981], although Duggal et al. were primarily interested in determining which subsets of stream-stream interaction regions and sector boundaries were responsible for statistically significant cosmic ray variations. As elegant as these earthoriented analyses are, they are nevertheless single-point measurements in which the reported latitudinal effects are very small compared to other temporal variations in intensity and for which the latitudinal distance to the current sheet is not directly measured and must be estimated.

Latitude variation of the galactic cosmic ray flux may also be investigated using the ratio of cosmic ray flux and observations of the current sheet at two spacecraft separated in latitude. Using Voyager 1 and 2 data from early 1981 through mid-1982, Decker et al. [1984] found a negligible latitudinal gradient with respect to the heliographic equator in the outer solar system. Our study of Voyager 1 and 2 data from late 1981 through mid-1983 complements the Decker et al. study by investigating the latitudinal organization of cosmic ray flux with respect to the heliospheric current sheet in the outer solar system. The model calculations in Figure 1 suggest that if two-point observations at low heliographic latitudes can be organized so one observation point is always closer to the current sheet than the other, then average gradients may be directly measured. In order to illustrate this approach, we first describe the analysis technique and apply it to model calculations of the intensity such as those in Figure 1. We then apply the same technique to the observations. A preliminary report of our results can be found in the article by Christon et al. [1985].

\section{Differential Measurement Technique}

As an illustration, assume that the cosmic ray flux at an observation point in the heliosphere is given by

$$
j_{i}=j_{b} \exp \left[G_{r}\left(r_{i}-R_{b}\right)\right] \exp \left\{G_{\Theta}\left[\Theta_{i}-\Theta_{c s}\left(r_{i}, \varphi_{i}\right)\right]\right\}
$$

where $G_{r}$ is the average radial component of the gradient, $G_{\boldsymbol{\theta}}$ 
is the colatitude component of the gradient, $R_{b}$ is the heliocentric distance to the boundary of the heliosphere, $j_{b}$ is the cosmic ray flux at $R_{b}$, assumed constant, $\Theta_{c s}(r, \varphi)$ is the colatitude of the current sheet, and $r, \varphi$, and $\Theta$ are the heliocentric distance, longitude, and colatitude of the point of observation, respectively. Colatitude $\Theta$ with the range $\left[0^{\circ}, 180^{\circ}\right]$ and latitude $\vartheta$ with the range $\left[90^{\circ} \mathrm{N}, 90^{\circ} \mathrm{S}\right]$ are related by $\vartheta=90^{\circ}-\Theta$. Magnetic latitude $\lambda_{m g}$ is related to colatitude by $\lambda_{m g}=\Theta_{c s}-\Theta$.

When a set of simultaneous observations is available at two or more well-separated points in interplanetary space, estimates of the latitudinal gradient can be obtained for each set of observations by selecting those observations when two spacecraft are on the same side of the current sheet and solving

$$
G_{\Theta}=\left[\ln \Gamma-G_{r}\left(r_{1}-r_{2}\right)\right] /\left(\Theta_{1}-\Theta_{2}\right)
$$

where $\Gamma$ is the ratio of the flux at Voyager 1 to the flux at Voyager 2. Warping of the heliospheric current sheet allows both spacecraft to sample the fluxes and therefore $G_{\boldsymbol{e}}$ in both magnetic hemispheres. Note that we have neglected the variation of $\Theta_{c s}$ with $r$ and $\varphi$ in calculating $G_{\Theta}$, since the true position of the current sheet $\Theta_{c s}$ is unknown in interplanetary space except at sector boundaries. Averages of $G_{\theta}$ are given by

$$
\left\langle G_{\Theta}\right\rangle=\left(\sum_{i} w_{G i} G_{\Theta \imath}\right) / \sum_{i} w_{G i}
$$

where $i$ runs from 1 to $n_{N N}$ or $n_{S s}$, i.e., is when the spacecraft are both north $(N N)$ or both south $(S S)$ of the current sheet, and $w_{G r}$ is the inverse square of $\sigma_{G_{\theta}}$, the uncertainty in $G_{\Theta i}$. The first letter of the two-character subscript identifies the magnetic field polarity (region) for Voyager 1, the second for Voyager 2 . We will show that on the average, $G_{\boldsymbol{\theta}}$, as defined above, yields useful information about intensity variations organized by the current sheet while removing the intensity variation due to $G_{r}$ and the varying separation in radial distance $r_{1}-r_{2}$.

Figure 2 shows a hypothetical heliospheric current sheet at a distance $r$ from the sun which is used to elucidate our measurement technique. As the current sheet corotates with the sun, the repeated sequences of a larger, longer wave or ripple followed by a smaller, shorter wave produce magnetic sectors of varying lengths at the representative spacecraft trajectories (horizontal lines). As a spacecraft passes through the current sheet, the longitudinal field component changes direction by $\sim 180^{\circ}$, the region of change being called an interplanetary sector boundary. Either spacecraft intercepting this current sheet would measure two longer sectors of opposite magnetic field polarity followed by two shorter sectors of opposite magnetic field polarity during each sequence, with the polarity determined by the magnetic hemispheres. The time from or until sector boundary crossings is a direct measure of the longitudinal distance of the spacecraft to the current sheet, and observed sector lengths are a direct measure of the longitudinal extent of waves in the current sheet.

In this study we explore the possibility that the sector lengths may also be an indication of the amplitude of the waves and therefore of the observer's latitudinal separation from the current sheet. For example, in Figure 2, Voyager 1 at point $A$ is as close to the current sheet azimuthally as at point $B$, but since the sector length at $B$ is much shorter than that at A, B may be closer to the current sheet in latitude than in longitude. Although the amplitude of the wavy current sheet is not necessarily smaller for shorter sectors, we will show below

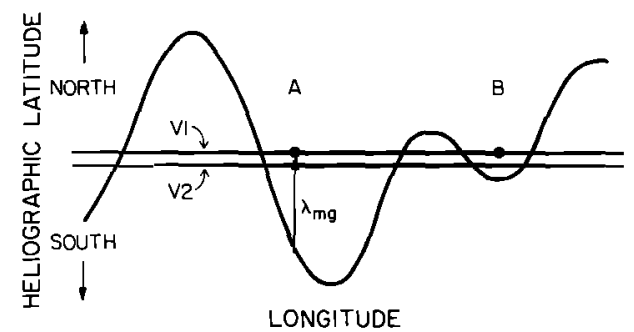

Fig. 2. Illustration of Voyager 1 (V1) and Voyager 2 (V2) trajectories and two sequences of recurring patterns in a hypothetical heliospheric current sheet. V1 is at equal azimuthal distances from the current sheet at points $A$ and $B$ but is closer to the current sheet at point $B$ than at point $A$.

that such a relationship appears to be consistent with our measurements.

Although the heliospheric current sheet in Figure 2 is represented as a simple combination of nearly sinusoidal curves, the topology of the heliospheric current sheet is more complex near the sun and is unknown in interplanetary space. For example, the near-sun current sheet at a distance of 2.5 solar radii, which is the footprint of the heliospheric current sheet, often has a more square wave than sine wave appearance with unequal north and south amplitudes during the period of this study [e.g., Hoeksema, 1984]. Though the general solar pattern may persist inside $1 \mathrm{AU}$, there is evidence for distortions of the current sheet beyond the near-sun region [e.g., Svalgaard and Wilcox, 1976; Seuss and Feynman, 1977; Tritakis, 1984]. In addition to probable near-sun distortions, interaction of solar wind streams beyond $1 \mathrm{AU}$ significantly modifies the interplanetary structure from that observed at $1 \mathrm{AU}$. Thus at present there is no way to determine the latitudinal distance of a given observation from the current sheet in the outer solar system, as was done by Newkirk et al. for observations at $1 \mathrm{AU}$. Moreover, the current sheet is not always thin even at $1 \mathrm{AU}$, and the transition through the boundary may last for hours or even days [see Klein and Burlaga, 1980; Behannon et al., 1983].

The latitudinal separation of our observations can, however, be utilized in combination with the magnetic field observations to extract general information about the cosmic ray spatial distribution. We use field polarities to ensure that both spacecraft are on the same side of the current sheet, sector lengths to order the measurements in terms of the latitudinal separation from the current sheet (latitudinal restriction), and times from sector boundary crossings to order the measurements in terms of the azimuthal separation from the current sheet (azimuthal restriction). We can therefore restrict our observations to those occasions in which one spacecraft is, on the average, closer to the current sheet than the other. As can be seen by comparing Figures 1 and 2, when both spacecraft are north of the current sheet at low heliographic latitudes, Voyager 1 will tend to observe a lower flux whenever Voyager 2 is closer to the current sheet, and when both are south at low heliographic latitudes, Voyager 2 will tend to observe a lower flux whenever Voyager 1 is closer. Restriction of observation positions and sectors in this manner qualitatively ensures that the observer longitudinally closer to the current sheet in a shorter sector is also more likely to be closer to the current sheet both in absolute angular distance and in latitudinal distance.

The signs of colatitude gradients should be opposite at symmetrical locations on either side of the current sheet in a drift-dominated transport regime. Therefore we evaluate $G_{\boldsymbol{A}}$, 
in which $G_{\Lambda_{N N}}=G_{\Theta_{N N}}$ and $G_{\Lambda_{s s}}=-G_{\mathbf{\theta}_{s s}}$. The average

$\left\langle G_{\Lambda}\right\rangle=\left(\sum_{i} w_{G i} G_{\Theta i}-\sum_{j} w_{G j} G_{\Theta j}\right) /\left(\sum_{i} w_{G i}+\sum_{j} w_{G j}\right)$

where $i(j)$ runs from 1 to $n_{N N}\left(n_{S S}\right)$ and $w_{G i(j)}$ is the inverse square of $\sigma_{G_{\theta_{r}(J)}}$, the uncertainty in $G_{\Theta_{1}(J)}$, should be independent of the detailed longitudinal structure of the current sheet $\left(\Theta_{c s}\right)$. With the aid of the numerical transport model we will demonstrate that the technique of calculating $\left\langle G_{\mathrm{A}}\right\rangle$, while placing restrictions on spacecraft positions relative to the current sheet, provides a useful diagnostic for investigating the distribution of cosmic rays in the heliosphere.

\section{A Transport Model With Current Sheet Organization}

The role of particle drift terms in the Fokker-Planck transport equation governing the propagation of galactic cosmic rays within the heliosphere has been explored theoretically only recently [see Kota and Jokipii, 1983, and references therein]. Diffusion enters into the model through the symmetric components of the diffusion tensor $\kappa$, which are assumed proportional to $K_{0} P^{1 / 2} \beta / B(r)$, where $K_{0}$ is the magnitude of the diffusion coefficient, $P$ is particle rigidity, $\beta$ is particle speed relative to the speed of light, and $B$ is the magnitude of the magnetic field [Jokipii and Davila, 1981]. Drift enters into the model through the antisymmetric components of $\kappa$, which are assumed proportional to $P \beta / B(r)$ [Jokipii et al., 1977]. These drift terms had not been included in previous evaluations of cosmic ray transport. The most recent refinement of calculations involving the drift terms [Kota and Joki$p i i, 1983]$ results in a three-dimensional, corotating, numerical model of the spatial variation of galactic cosmic ray flux. In this model, cosmic ray transport is strongly influenced by the drift terms, and the flux is roughly symmetric about the heliographic poles and organized by the heliospheric current sheet. The isointensity contours in Figure 1 resulting from this model are qualitatively representative of the model solutions for $\sim 1.6-\mathrm{GeV}$ protons (rigidity $P=2.3 \mathrm{GV}$ ) throughout the region of our investigation.

In the model calculations for the current phase of the 22year cycle, particles diffuse down in latitude from the poles and drift and diffuse upward in latitude from the current sheet, resulting in intensity minima away from the current sheet and gradients at low heliographic latitudes of opposite signs in opposite sectors. Although the gradient depends on both the heliographic latitude and the magnitude of the diffusion coefficient, at low heliographic latitudes the intensity in a given sector tends to be higher nearer the current sheet.

In an attempt to focus on model predictions appropriate to our situation, i.e., one in which the true separation from the current sheet is not well known but in which the longitudinal distance from current sheet encounters is known, we use the following procedure. The flux is evaluated at points equally spaced $\left(10^{\circ}\right)$ in longitude between the current sheet and equally spaced $\left(5^{\circ}\right)$ in latitude. Results are obtained in the latitude range bounded above by $20^{\circ} \mathrm{N}$ and below by $4^{\circ} \mathrm{S}$, the extremes of Voyager latitudes during this period. We calculate the colatitude gradient $G_{\Theta}{ }^{*}=\ln \Gamma / \Delta \Theta$ from these model predictions, where $\Gamma$ is the ratio of the flux at the more northerly point (Voyager 1) to that at the more southerly point (Voyager 2) and $\Delta \Theta$ is a representative colatitudinal spacecraft separation. Expectation values $\left\langle G_{\theta}{ }^{*}\right\rangle$, relatively independent of the relative longitudinal positions of the spacecraft, are calculated via equation (3), using equal uncertainties by averaging over all possible combinations of observation points while keeping the sign and magnitude of the colatitudinal separation

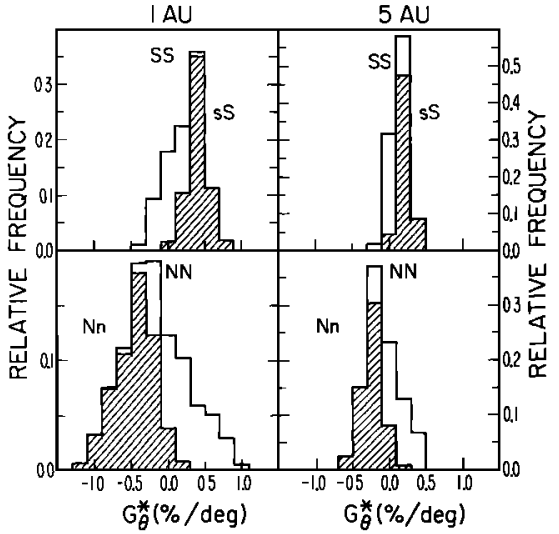

Fig. 3. Predictions of the distribution of $G_{\theta}$, the colatitude gradient, at observation points separated by $\sim 15^{\circ}$ in a latitude band from $20^{\circ} \mathrm{N}$ to $4^{\circ} \mathrm{S}$ for the intensity contours in Figure 1. The histograms including both the shaded and unshaded portions are distributions of unrestricted data whose averages at $1 \mathrm{AU}$ are $\left\langle G_{\boldsymbol{Q}}{ }^{*}\right\rangle_{N N}=-0.18$ \pm 0.02 and $\left\langle G_{\theta}{ }^{*}\right\rangle_{S S}=+0.25 \pm 0.01 \% / \mathrm{deg}$ and at $5 \mathrm{AU}$ are $\left\langle G_{\Theta}{ }^{*}\right\rangle_{N N}=-0.10 \pm 0.01$ and $\left\langle\bar{G}_{\theta}{ }^{*}\right\rangle_{S S}=+0.14 \pm 0.01 \% / \mathrm{deg}$. The shaded portions are the azimuthally restricted subsets whose averages at $1 \mathrm{AU}$ are $\left\langle G_{\Theta}{ }^{*}\right\rangle_{N_{n}}{ }^{a z}=-0.45 \pm 0.02$ and $\left\langle G_{\theta}{ }^{*}\right\rangle_{\mathrm{ss}}{ }^{a z}=+0.40$ $\pm 0.01 \% / \mathrm{deg}$ and at $5 \mathrm{AU}$ are $\left\langle G_{\theta}{ }^{*}\right\rangle_{N n}{ }^{{ }^{z}}=-0.24 \pm 0.01$ and $\left\langle G_{\mathrm{\theta}}{ }^{*}\right\rangle_{\mathrm{ss}}{ }^{a z}=+0.21 \pm 0.01 \% / \mathrm{deg}$.

fixed. This simulates the actual experimental situation to some degree. Since Voyager 1 is at north latitudes and Voyager 2 is near the equator, one generally expects $G_{\theta}<0$ when both spacecraft are north of the current sheet and $G_{\theta}>0$ when both spacecraft are south of it.

As an illustrative example we have analyzed the model calculations corresponding to Figures $1 a$ and $1 b$ for observations at 1 and $5 \mathrm{AU}$, where $K_{0}=5 \times 10^{21} \mathrm{~cm}^{2} \mathrm{~s}^{-1}, R_{b}=10 \mathrm{AU}$ and $\alpha=30^{\circ}$, a set of model parameters in which the effects of drift are clearly important. A representative spacecraft separation of $15^{\circ}$ is chosen. The histograms in Figure 3, including both the shaded and unshaded portions, represent the unrestricted sampling of $G_{\Theta}{ }^{*}$ for $N N$ and $S S$ model predictions and show that $\left\langle G_{\Theta}{ }^{*}\right\rangle_{N N}$ and $\left\langle G_{\Theta}{ }^{*}\right\rangle_{S S}$ (listed in the figure caption) are of opposite sign and similar magnitude. The $N N$ and $S S$ subsets have different averages and modes because of the latitude gradient, so that the unrestricted average calculated from equation (4) is $\left\langle G_{\mathrm{A}}^{*}\right\rangle=-0.20 \pm 0.01 \% / \mathrm{deg}$ at 1 AU and $\left\langle G_{\Lambda}{ }^{*}\right\rangle=-0.11 \pm 0.01 \% / \mathrm{deg}$ at $5 \mathrm{AU}$. The negative sign of $\left\langle G_{\Lambda}{ }^{*}\right\rangle$ indicates that the flux generally decreases with increasing latitude separation from the current sheet in the region sampled.

Restriction of the azimuthal positions of the observation points in the manner described in the previous section selects the subsets of observations indicated by the shaded distributions in Figure 3. The lowercase letter in the two-character identifier indicates the observation point closer to the current sheet. Note that the averages (listed in the figure caption) and modes of these azimuthally restricted data are more clearly separated than they were for the unrestricted data. The superscript indicates the type of selection criterion applied. The azimuthally restricted average at $1 \mathrm{AU}$ is $\left\langle G_{\Lambda}{ }^{*}\right\rangle^{\alpha z}=-0.44$ $\pm 0.01 \% / \mathrm{deg}$ and at $5 \mathrm{AU}$ is $\left\langle G_{\Lambda}{ }^{*}\right\rangle^{a z}=-0.23 \pm 0.01 \% / \mathrm{deg}$, showing a factor of 2 enhancement of $\left\langle G_{\Lambda}{ }^{*}\right\rangle$ at both sampling radii due to the application of the azimuthal restriction. Note that because of the simple sinusoidal form of the model current sheet the latitudinal restrictions discussed previously are neither applicable nor necessary.

The model calculations depend on a number of parameters, 
TABLE 1. Model Predictions of $\left\langle G_{\Lambda}{ }^{*}\right\rangle$

\begin{tabular}{|c|c|c|c|c|c|c|c|}
\hline \multirow{3}{*}{$\begin{array}{l}\Delta \Theta \\
\operatorname{deg}\end{array}$} & \multicolumn{3}{|c|}{ Model Parameters } & \multirow{2}{*}{\multicolumn{2}{|c|}{$\left\langle G_{\Lambda}{ }^{*}\right\rangle, \% /$ deg }} & \multirow{2}{*}{\multicolumn{2}{|c|}{$\left\langle G_{\Lambda}{ }^{*}\right\rangle^{a z}, \% / \mathrm{deg}$}} \\
\hline & & & & & & & \\
\hline & $\mathrm{cm}^{2} \mathrm{~s}^{-1}$ & $\mathrm{AU}$ & deg & $1 \mathrm{AU}$ & $5 \mathrm{AU}$ & $1 \mathrm{AU}$ & $5 \mathrm{AU}$ \\
\hline \multirow[t]{2}{*}{15} & $5.0 \times 10^{21}$ & 10 & $\begin{array}{l}30 \\
45 \\
60\end{array}$ & $\begin{array}{l}-0.20 \\
-0.16 \\
-0.17\end{array}$ & $\begin{array}{l}-0.11 \\
-0.07 \\
-0.05\end{array}$ & $\begin{array}{l}-0.44 \\
-0.48 \\
-0.49\end{array}$ & $\begin{array}{l}-0.23 \\
-0.20 \\
-0.18\end{array}$ \\
\hline & $1.5 \times 10^{22}$ & 15 & $\begin{array}{l}30 \\
45 \\
60\end{array}$ & $\begin{array}{l}-0.02 \\
-0.02 \\
-0.04\end{array}$ & $\begin{array}{l}-0.02 \\
-0.01 \\
-0.01\end{array}$ & $\begin{array}{l}-0.09 \\
-0.11 \\
-0.14\end{array}$ & $\begin{array}{l}-0.05 \\
-0.04 \\
-0.04\end{array}$ \\
\hline \multirow[t]{2}{*}{20} & $5.0 \times 10^{21}$ & 10 & $\begin{array}{l}30 \\
45 \\
60\end{array}$ & $\begin{array}{l}-0.19 \\
-0.15 \\
-0.16\end{array}$ & $\begin{array}{l}-0.11 \\
-0.06 \\
-0.05\end{array}$ & $\begin{array}{l}-0.36 \\
-0.38 \\
-0.40\end{array}$ & $\begin{array}{l}-0.19 \\
-0.16 \\
-0.14\end{array}$ \\
\hline & $1.5 \times 10^{22}$ & 15 & $\begin{array}{l}30 \\
45 \\
60\end{array}$ & $\begin{array}{l}-0.02 \\
-0.02 \\
-0.03\end{array}$ & $\begin{array}{l}-0.02 \\
-0.01 \\
-0.01\end{array}$ & $\begin{array}{l}-0.07 \\
-0.08 \\
-0.11\end{array}$ & $\begin{array}{l}-0.04 \\
-0.03 \\
-0.03\end{array}$ \\
\hline
\end{tabular}

such as tilt angle $\alpha$, diffusion coefficient $K_{0}$, and distance of the outer boundary $R_{b}$, for example. These parameters are responsible for the general differences between the model predictions in Figures $1 a$ and $1 b$ and those in Figures $1 c$ and $1 d$. Sampling parameters such as the radial distance $r$, spacecraft latitude separation $\Delta \Theta$, and latitude band sampled also produce differences in the values of $\left\langle G_{\Lambda}{ }^{*}\right\rangle$ obtained from each set of model parameters. The effects of varying the model parameters and sampling parameters are demonstrated by intercomparing the values of $\left\langle G_{\Lambda}{ }^{*}\right\rangle$ derived from the four different sets of model parameters listed in Table 1 for various choices of sampling parameters. Relative uncertainties in the model predictions in Table 1 are always $\lesssim 0.25$ and $\$ 0.06$ for unrestricted and azimuthally restricted subsets, respectively, and are suppressed in the Table for clarity and ease of reading. Because of the unrealistically small values of $R_{b}$ used here, the predictions at $5 \mathrm{AU}$ merely suggest conditions expected well beyond $1 \mathrm{AU}$ but not yet close to the boundary of the heliosphere and should not be interpreted as specific predictions of cosmic ray intensity variations at $5 \mathrm{AU}$. However, in all of the model and sampling situations displayed in Table 1 one major factor is apparent: application of the azimuthal restriction results in a larger, more negative value of $\left\langle G_{\Lambda}^{*}\right\rangle$, reflecting a general decrease in cosmic ray flux with increasing separation from the current sheet.

The effects of varying the modeling and sampling parameters are demonstrated by comparing the values of $\left\langle G_{\Lambda^{*}}{ }^{*}\right\rangle$ in Table 1. For example, values of $\left\langle G_{\Lambda}{ }^{*}\right\rangle$ for $K_{0}=5 \times 10^{21} \mathrm{~cm}^{2}$ $\mathrm{s}^{-1}$ are larger than those for $K_{0}=1.5 \times 10^{22} \mathrm{~cm}^{2} \mathrm{~s}^{-1}$, as might be expected. The model also yields increased values of $\left\langle G_{\Lambda}{ }^{*}\right\rangle^{a z}$ at $1 \mathrm{AU}$ for larger current sheet tilts, but slightly decreased values at $5 \mathrm{AU}$, implying that at low heliographic latitudes, $\left\langle G_{\Lambda}{ }^{*}\right\rangle^{a z}$ is not strongly dependent on $\alpha$, which is unknown in the experimental situation. Our analysis of the model also predicts systematically smaller values of $\left\langle G_{\Lambda}^{*}\right\rangle$ and $\left\langle G_{\Lambda}{ }^{*}\right\rangle^{a z}$ at $5 \mathrm{AU}$ than at $1 \mathrm{AU}$, suggesting that observations in the inner and outer heliosphere may differ. Finally, differences in $\left\langle G_{\Lambda}{ }^{*}\right\rangle$ and $\left\langle G_{\Lambda}{ }^{*}\right\rangle^{a z}$ due to differences in latitude separation are small, suggesting that the accumulation of data from an extended period will not compromise our ability to discern important trends in $G_{\Lambda}$.

\section{Data Selection}

This study covers the period from day 240,1981 , until day 190,1983 , during which the separation of the Voyagers increased from 1.9 to $4.7 \mathrm{AU}$ in radial distance from the sun (Voyager 1 traveled from 11 to $17 \mathrm{AU}$ ) and from $10^{\circ}$ to $21^{\circ}$ in latitude (Voyager 1 traveled from $6^{\circ} \mathrm{N}$ to $20^{\circ} \mathrm{N}$ ). The Voyagers were separated by $\leq 16^{\circ}$ in longitude. Because of the separation in longitude and radius the probability that the spacecraft are both in the same magnetic sector during this period is low.

In order to determine the instantaneous radial and latitudinal gradients represented in Figure 1, daily samples of the counting rates of protons with kinetic energies of $>75 \mathrm{MeV}$ and a median energy of $\sim 0.95 \mathrm{GeV}(\sim 1.6-\mathrm{GV}$ rigidity) measured by the nearly identical High Energy Telescopes of the Cosmic Ray Subsystem on the Voyager 1 and 2 spacecraft [Stone et al., 1977] were compared (see Figure 4). Ratios of the Voyager 1 to Voyager 2 counting rates, adjusted for the difference in geometrical factors of the telescopes [Cummings and Webber, 1983], are plotted in the upper panel of Figure 4. The relative uncertainty in this adjustment is $\sim 0.2 \%$ and is applied identically to all values of $\Gamma$. Uncertainties in

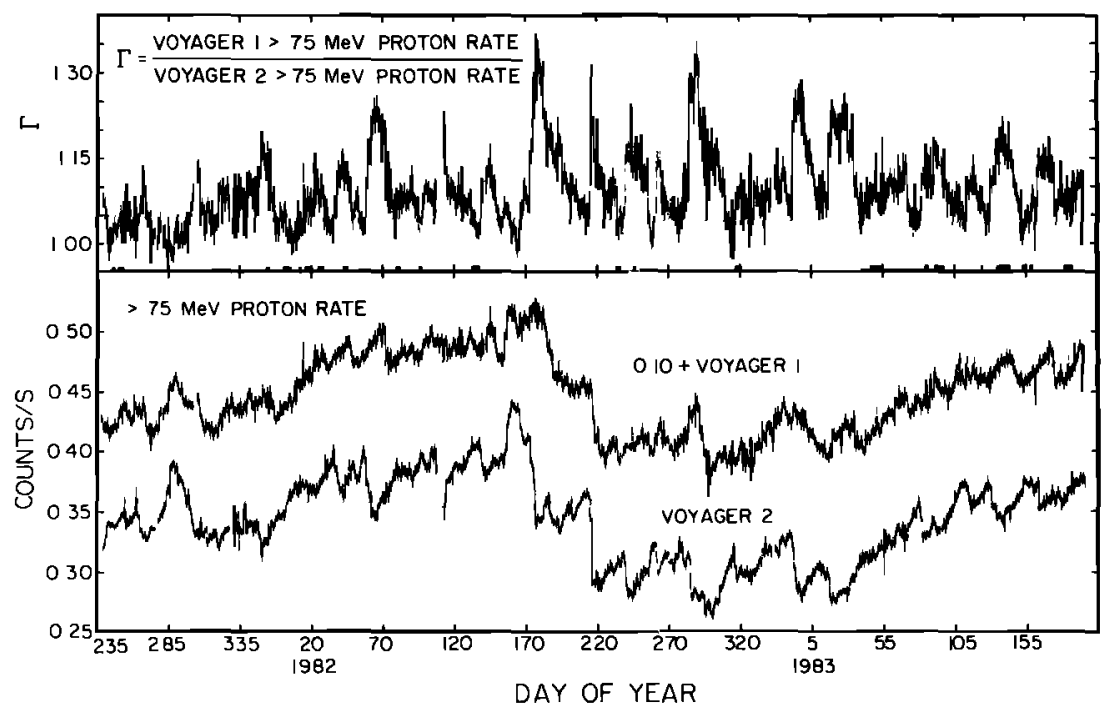

Fig. 4. (Top) The ratio of daily samples of the Voyager 1 to the Voyager 2 counting rate of $>75-\mathrm{MeV}$ protons. The ratios have been adjusted for a difference in geometrical factors of the telescopes. Underscored periods indicate data used in this study to estimate the latitude gradient. (Bottom) Daily samples of the counting rates of $>75-\mathrm{MeV}$ protons from Voyagers 1 and 2. Voyager 1 is offset for clarity. 


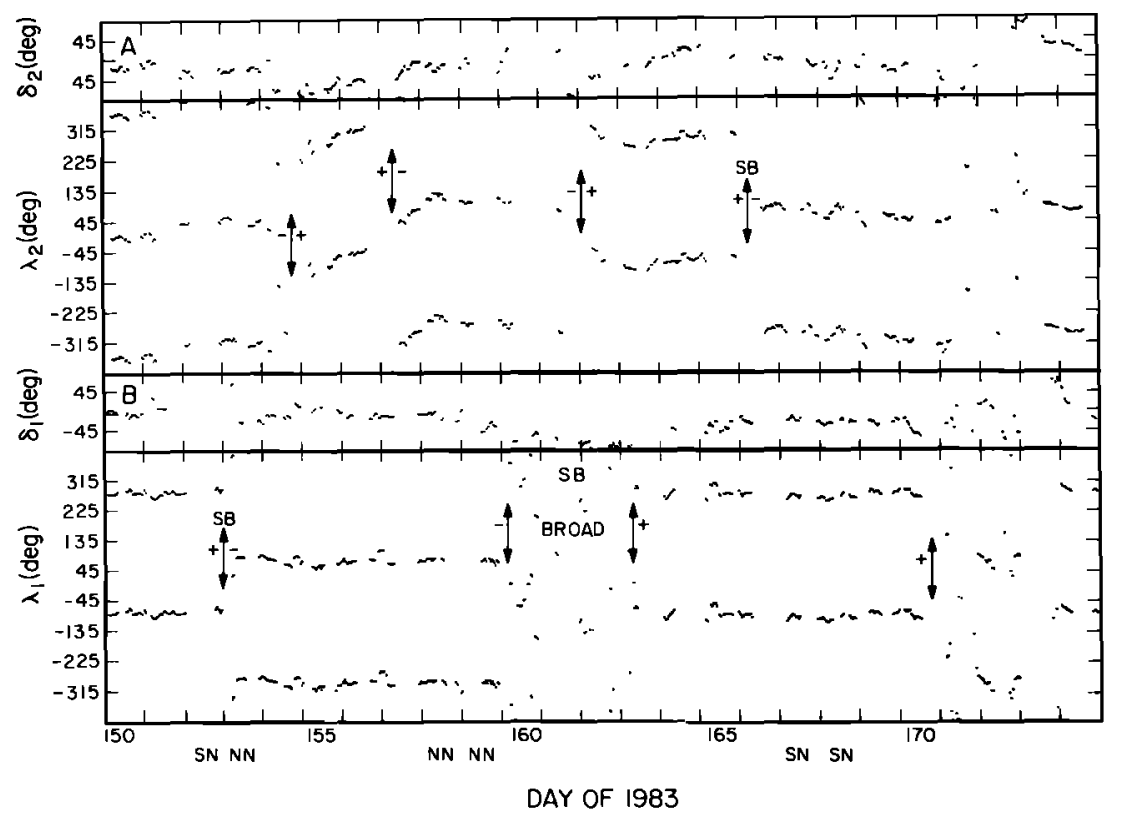

Fig. 5. Magnetic field azimuth and elevation angles from Voyagers 1 and 2 . The field azimuth, $\lambda$, is $0^{\circ}$ when the field is directed away from the sun. The field elevation, $\delta$, is $0^{\circ}$ when the field is along the spacecraft-sun line. Two cycles are plotted for $\lambda$ so that sector boundaries $\left(\sim 180^{\circ}\right.$ changes) and turbulent field periods are more easily separated. Doubleheaded arrows highlight changes which are interpreted as penetrations of the current sheet. Those arrows labeled with "SB" are most similar to sector boundaries near earth. Below: days labeled with SN or NN are included in this study.

Figure 4 are due to counting statistics only. The maximum and mean relative uncertainties due to counting statistics in the daily counting rate samples used are $1.7 \%$ and $1.1 \%$, respectively. Temporal variations of the energy spectra of lowerenergy protons as well as the abundance ratios of lowerenergy nuclei from carbon to iron were used to eliminate periods with probable solar flare contributions.

From late 1981 until late 1982 the cosmic ray fluxes are dominated by three rather long ( $\approx 100$-day) periods during which the overall flux levels neither increase nor decrease appreciably with time. A moderate recovery for $\sim 50$ days at the start of 1982 separates the late 1981 and early 1982 level flux periods. Dramatic flux decreases (Forbush decreases) in mid1982 mark the passage of solar flare shocks and transient material. At the start of 1983 the fluxes at both Voyagers start to increase steadily with time as they continue to recover from solar modulation.

Hourly averages of the interplanetary magnetic field azimuth and elevation angles measured by the Voyager Magnetic Field Experiment [Behannon et al., 1977] are used in this study. Figure 5 is an example of the magnetic field data plots which were used to determine the sector polarities, sector boundaries, and periods of field turbulence.

A magnetic sector, for use in this study, is required to be a time period greater than 4.5 days during which the field azimuth remains predominantly within $90^{\circ}$ of the expected spiral field direction leading either toward the sun (negative, shown as a minus sign on the figure) or away from the sun (positive, shown as a plus sign). The expected field azimuth is $\sim 85^{\circ}$ at the Voyagers for this time period, and the expected elevation is within $\sim 45^{\circ}$ of the heliographic equator. During the present portion of the 22-year magnetic activity solar cycle, negative fields are associated with the predominant polarity of the sun's north rotational pole, and positive fields are associated with the sun's south rotational pole. (Below, $N$ and $S$ will be used to denote negative and positive polarity fields, respectively). Although shorter magnetic sectors exist, the longer magnetic sectors ( $\geq 4.5$ days) most probably result from plasma flow from the larger near-equatorial coronal holes that are often linked to the sun's poles. A sector boundary is here defined to be at the centroid of the transition region from adjacent sectors of opposite polarities. If a sector boundary occurs within a data gap $\leq 1$ day long, the center of the gap was taken to be the time of the sector boundary. Sector boundaries occurring in longer data gaps were not included.

Data days were first selected by a computer search through lists of the field azimuth, in which calendar days were accepted for analysis if $\mathbf{0 . 7 5}$ of those hourly averages collected fell within the current magnetic sector. Subsequently, detailed plots similar to Figure 5 were scanned to assure that the selection is valid and that there are $\gtrsim 8$ hours of usable magnetic field data at each Voyager, and to determine the length of the sectors in which the data day appeared. This selection procedure is analogous to those used in similar analyses of sector structure [e.g., Klein and Burlaga, 1980; Hoeksema 1984]. Some of the sector boundaries shown in Figure 5 (double-headed vertical arrows) appear as sharp divisions between fields of opposite polarity. These sharp divisions, labeled with "SB," are typical of what one might call "classical" sector boundaries observed at $1 \mathrm{AU}$. At other boundaries the separation of sectors is not so clear, but the fields at \pm 2 days from the boundary are often definitely of opposite polarities. Extremely turbulent periods, for example, from day 160 to 163 and after day 170 (see Figure 5), are not included in the data sets. The turbulent periods are most probably due to solar transients propagating through the region.

\section{Data Set Selection}

Criteria for the initial data collection, as detailed above, are: (1) the spacecraft were required to be completely in a region of magnetic field pointing either generally toward or away from the sun; (2) all periods with probable contamination from solar flare particles were removed; and (3) all periods surrounding Forbush decreases, such as those encountered on 
$\sim 175,1982$, and 220,1982 , were eliminated. This produced data set I (DS I), which includes the subsets $N N, S S, N S$, and $S N$. Data subsets $N S$ and $S N$ are used below as an independent data set in order to determine the radial gradient. Following this selection procedure, $\sim 22 \%$ of our total data remained, there being a total of $\mathbf{4 2}$ magnetic sectors at Voyagers 1 and 2 that are used in $16 N N$ and $7 \mathrm{SS}$ combinations. Proceeding with more restrictive selection criteria, we eliminated from the DS I $N N$ and $S S$ subsets the days in which segments of sectors were not recognizable, i.e., days with large fluctuations in field direction, with systematic departures from the spiral direction in azimuth or elevation, or with insufficient data. The resulting subset of DS I data retained for analysis is called DS II. DS II was divided into two subsets: DS II $a$, in which sectors were clearly defined, and DS II $b$, in which sectors might have been present but were disturbed or distorted. Specifically, in DS II $a$, the magnetic field direction was uniform and close to the expected spiral direction, whereas on days in DS II $b$ the magnetic field direction fluctuated somewhat or showed systematic deviations from the spiral direction.

\section{Azimuthal and Latitudinal Restrictions}

Restrictions are applied so that on the average, in the north, Voyager 2 is expected to be closer to the current sheet than Voyager $1(\mathrm{Nn})$, and in the south, Voyager 1 is expected to be closer to the current sheet than Voyager $2(s S)$. Specifically, the azimuthal restriction (az) is applied by averaging only those days during which Voyager 2 (1) is closer in time from the current sheet than Voyager 1 (2) (corresponding to a smaller azimuthal separation) in north (south) polarity fields. The latitudinal restriction (lat) is applied by averaging only those days during which the sector length at Voyager 1 (2) is longer than that at Voyager 2 (1) (corresponding to a greater latitudinal separation) in north (south) polarity fields.

\section{Results AND Discussion}

\section{The Radial Gradient}

Determination of $\left\langle G_{\Lambda}\right\rangle$ requires knowledge of the value of $G_{r}$, which can be estimated directly from the $N S$ and $S N$ subsets, since latitude variations having opposite signs should cancel on the average in either of these subsets. $G_{r}$ can also be determined from the $N N$ and $S S$ data sets analyzed in combination, since the latitude gradients, which have opposite signs in the magnetic hemispheres above and below the current sheet, should average to zero in combination. We use DS I subsets to calculate our estimate of the radial gradient

$$
\left\langle G_{r}\right\rangle=\left[\sum_{i} w_{\Gamma_{i}} \ln \Gamma_{i} /\left(r_{1 i}-r_{2 i}\right)\right] / \sum_{i} w_{\Gamma_{i}}
$$

where $i$ runs from 1 to the number of observations in the subset and $w_{\Gamma i}$ is the inverse square of $\sigma_{\Gamma i}$, the uncertainty in $\Gamma_{t}$. Distributions of the daily estimates $G_{r i}=\ln \Gamma_{i} /\left(r_{1 i}-r_{2 t}\right)$ for the $N S, S N, N N$, and $S S$ subsets plotted in Figure 6 show that the most likely value of $\left\langle G_{r}\right\rangle$ is $\sim 2 \%$ /AU, consistent with the value obtained by Decker et al. [1984], and that any splitting of these subsets due to latitude variations is not apparent. Note that $N S, S N, N N$, and $S S$ are independent data sets. The two combinations $N S, S N$ and $N N, S S$ are likewise independent data. Our estimates of $G$, calculated from the combined $N S$ and $S N$ subsets, $\left\langle G_{r}\right\rangle_{N S, S N}=2.21 \pm 0.11 \% / \mathrm{AU}$, and from the combined $N N$ and $S S$ subsets, $\left\langle G_{r}\right\rangle_{N N, S S}=2.29$ $\pm 0.13 \% / \mathrm{AU}$, are consistent, suggesting that the best estimate is an average over all 152 days of data combined (bottom

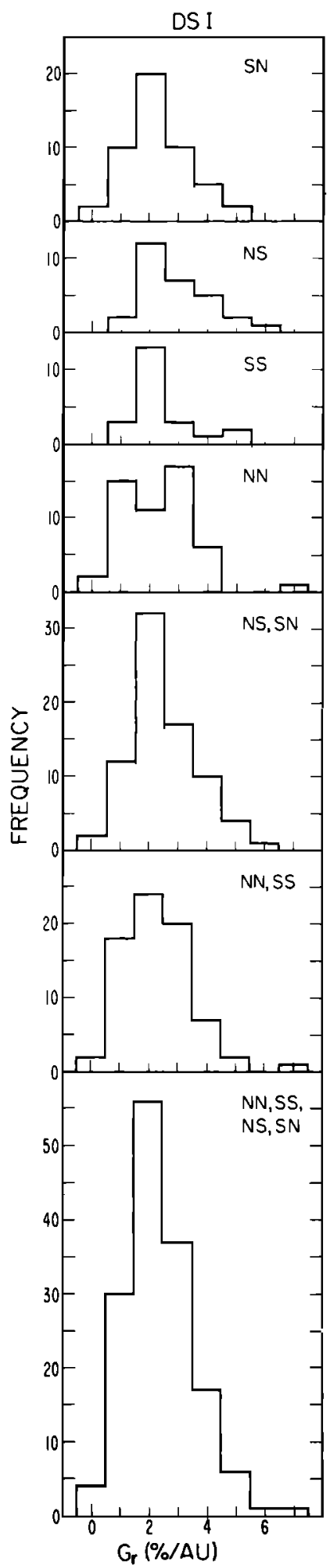

Fig. 6. $G_{r}$ for subsets of DS I.

panel of Figure 6), which yields $\left\langle G_{r}\right\rangle=2.25 \pm 0.08 \% / \mathrm{AU}$. Our estimate $\left\langle G_{r}\right\rangle$, compared to other recent estimates of $G_{r}$ in Table 2, shows the greatest similarity to the values obtained by Decker et al. [1984], over nearly the same time interval as ours, and by Van Allen and Randall [1985], over many years of data. Note that in all except two of the radial gradient studies listed in Table 2, observation times have been adjusted for a delay due to propagation of information with the solar 
TABLE 2. Recent Estimates of Cosmic Ray Radial Gradient

\begin{tabular}{|c|c|c|c|c|c|}
\hline Study & $\begin{array}{l}\text { Averaging } \\
\text { Period, } \\
\text { Days }\end{array}$ & $\begin{array}{l}\text { Spacecraft } \\
\text { Used }\end{array}$ & $\begin{array}{l}V_{\text {shift }} \text {, } \\
\mathrm{km} / \mathrm{s}\end{array}$ & $\begin{array}{l}\text { Time } \\
\text { Period }\end{array}$ & $\begin{array}{c}G_{r}, \\
\% / A U\end{array}$ \\
\hline $\begin{array}{l}\text { Lockwood and } \\
\text { Webber }\end{array}$ & 26 & V1, V2, P10, IMP 8 & $(800,400, \infty)^{b}$ & $1975-1982$ & $3.0 \pm 0.3$ \\
\hline Venkatesan et al. [1984] & 27 & V1, V2, IMP 8 & 500 and $\infty$ & $1977-1982$ & $2-4$ \\
\hline Decker et al. [1984] & 26 & $\mathrm{~V} 1, \mathrm{~V} 2$ & $\infty$ & $1981-1982$ & $2^{c}$ \\
\hline Venkatesan et al. [1985] & 27 & V1, V2, P10, P11, IMP 8 & 500 & $1977-1982$ & 3 \\
\hline $\begin{array}{l}\text { Van Allen and } \\
\text { Randall }[1985]\end{array}$ & 25 & P10, P11 & $\approx 440^{d}$ & $1972-1984$ & $2.06 \pm 0.20$ \\
\hline This study & 1 & V1, V2 & $\infty$ & $1981-1983$ & $2.25 \pm 0.08$ \\
\hline
\end{tabular}

Measurements of cosmic rays with kinetic energies $\gtrsim 70 \mathrm{MeV}$.

$a_{\infty}$ : no shift corresponds to an instantaneous propagation speed.

'Three different values intermixed.

${ }^{c}$ Either $G_{r}=2 \% / \mathrm{AU}$ assuming $G_{3}=0$ or $G_{3}=0.4 \% / \mathrm{deg}$ assuming $G_{r}=0$.

${ }^{d}$ Values range from 250 to 750 .

wind before analysis. In addition to the instantaneous analysis which forms the basis of this paper, we have also performed a "shifted" analysis of the early 1983 data and find that the shifted value of $\left\langle G_{r}\right\rangle$ is $2.24 \pm 0.09 \%$ /AU. Inspection of equations (2) and (3) reveals that the average dependence of $\left\langle G_{\theta}\right\rangle$ on $G_{r}$ is $\sim\left\langle r_{1}-r_{2}\right\rangle\left\langle\left\langle\Theta_{1}-\Theta_{2}\right\rangle\right.$, so that an uncertainty of $0.1 \% / \mathrm{AU}$ in the assumed $G_{r}$ introduces an error of only $0.02 \% / \mathrm{deg}$ in the derived $\left\langle G_{\Theta}\right\rangle$.

\section{Latitude Variations}

Figure 7 shows the distributions of $G_{\theta}$ corrected for $G_{r}=$ $2.25 \% / \mathrm{AU}$ for DS I subsets $S N, N S, S S$, and $N N$. The modes of these distributions are generally centered on $G_{\theta}=0$. In addition, the $s S^{a z}$ and $N n^{a z}$ DS I subsets show only a very small splitting due to a latitudinal gradient, as might be expected, since in this data set we have not been selective about the presence of turbulence or the structure of the boundaries between magnetic sectors. Histograms of the distributions of $G_{\theta}$ for DS II unrestricted, azimuthally restricted, latitudinally restricted, and latitudinally and azimuthally restricted subsets are plotted in Figure 8. Average values appear in Table 3 and may be qualitatively compared to the model distributions in Figure 3 , since similar restrictions have been applied to data and model predictions. Note that once the spacecraft positions are restricted either azimuthally or latitudinally, the averages and modes of the $N n G_{\mathbf{e}}$ distributions shift to generally more negative values, a trend consistent with our analysis of the model calculations.

Figure 9 shows the distributions of $G_{\wedge}$ for DS II. Average values appear in Table 3. Unrestricted averages are consistent with no latitude gradient, while restricted averages are statistically significant and negative. Since differences between $\left\langle G_{\Lambda}\right\rangle$ for DS II $a$ and DS II $b$ are statistically insignificant in all cases, we may conclude that moderate deviations or fluctuations of the magnetic field direction from the spiral direction do not significantly affect these values of $\left\langle G_{\Lambda}\right\rangle$, and we therefore consider the $\left\langle G_{\Lambda}\right\rangle$ for DS II as our best estimates in restricted and unrestricted subsets. The trend displayed in Table 3 through the application of restrictions to our data is consistent with the trend displayed in Table 1 through the application of restrictions to the model. Both trends indicate a decrease in cosmic ray flux with increasing distance from the current sheet. Note, however, that at $5 \mathrm{AU}$, (1) for unrestricted data the values of $\left\langle G_{\Lambda}\right\rangle$ are consistent with $\left\langle G_{\Lambda}{ }^{*}\right\rangle$ for $K_{0}=$ $1.5 \times 10^{22} \mathrm{~cm}^{2} \mathrm{~s}^{-1}$ but smaller than $\left\langle G_{\Lambda}{ }^{*}\right\rangle$ for $K_{0}=5 \times 10^{21}$ $\mathrm{cm}^{2} \mathrm{~s}^{-1}$, and (2) for azimuthally restricted data, the values of

TABLE 3. $\left\langle G_{\boldsymbol{\theta}}\right\rangle$ and $\left\langle G_{\boldsymbol{A}}\right\rangle$

\begin{tabular}{|c|c|c|c|c|}
\hline & \multicolumn{4}{|c|}{ Data Sets } \\
\hline & I & II & II $a$ & II $b$ \\
\hline $\begin{array}{l}\left\langle G_{\boldsymbol{\Theta}}\right\rangle_{N N} \\
\left\langle G_{\boldsymbol{\theta}}\right\rangle_{S S} \\
\left\langle G_{\boldsymbol{\Lambda}}\right\rangle\end{array}$ & $\begin{array}{r}0.02 \pm 0.03(52) \\
-0.02 \pm 0.04(22) \\
0.02 \pm 0.03(74)\end{array}$ & $\begin{array}{r}\text { Unrestricted Ave } \\
0.03 \pm 0.04(29) \\
-0.01 \pm 0.04(19) \\
0.02 \pm 0.03(48)\end{array}$ & $\begin{array}{l}{ }^{e}-0.02 \pm 0.05(21) \\
-0.06 \pm 0.03(6) \\
-0.01 \pm 0.04(27)\end{array}$ & $\begin{array}{l}0.14 \pm 0.09(8) \\
0.01 \pm 0.05(13) \\
0.06 \pm 0.05(21)\end{array}$ \\
\hline $\begin{array}{l}\left\langle G_{\Theta}\right\rangle_{N n^{a z}}^{a z} \\
\left\langle G_{\Theta}\right\rangle_{s S}^{a z} \\
\left\langle G_{\Lambda}\right\rangle^{a z}\end{array}$ & $\begin{array}{c}-0.12 \pm 0.04(21) \\
0.09 \pm 0.07(7) \\
-0.11 \pm 0.03(28)\end{array}$ & $\begin{array}{c}\text { imuthally Restricteo } \\
-0.18 \pm 0.05(10) \\
0.12 \pm 0.09 \text { (5) } \\
-0.16 \pm 0.04(15)\end{array}$ & $\begin{array}{l}\text { verage } \\
\qquad-0.18 \pm 0.05(10) \\
-0.06 \pm 0.08(1) \\
-0.15 \pm 0.05(11)\end{array}$ & $\begin{array}{c}\cdots(0) \\
0.20 \pm 0.09(4) \\
-0.20 \pm 0.09(4)\end{array}$ \\
\hline 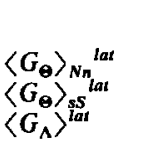 & $\begin{array}{c}-0.15 \pm 0.04(24) \\
0.09 \pm 0.13(2) \\
-0.15 \pm 0.04(26)\end{array}$ & $\begin{array}{l}\text { titudinally Restricte } \\
-0.22 \pm 0.03(11) \\
0.09 \pm 0.13(2) \\
-0.20 \pm 0.03(13)\end{array}$ & $\begin{array}{l}\text { verage } \\
\qquad \begin{aligned}-0.21 \pm 0.03(10) \\
\cdots(0) \\
-0.21 \pm 0.03(10)\end{aligned}\end{array}$ & $\begin{array}{r}-0.30 \pm 0.09(1) \\
0.09 \pm 0.13(2) \\
-0.15 \pm 0.10(3)\end{array}$ \\
\hline 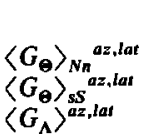 & $\begin{array}{c}\text { Azimuth } \\
-0.16 \pm 0.04(17) \\
0.23 \pm 0.08(1) \\
-0.16 \pm 0.04(18)\end{array}$ & $\begin{array}{r}\text { and Latitudinally } F \\
-0.22 \pm 0.04(8) \\
0.23 \pm 0.08(1) \\
-0.22 \pm 0.03(9)\end{array}$ & $\begin{array}{l}\text { tricted Average } \\
-0.22 \pm 0.04(8) \\
\cdots(0) \\
-0.22 \pm 0.04(8)\end{array}$ & $\begin{array}{c}\cdots(0) \\
0.23 \pm 0.08(1) \\
-0.23 \pm 0.08(1)\end{array}$ \\
\hline
\end{tabular}

Values are in \%/deg. Numbers in parentheses are days of observation. 


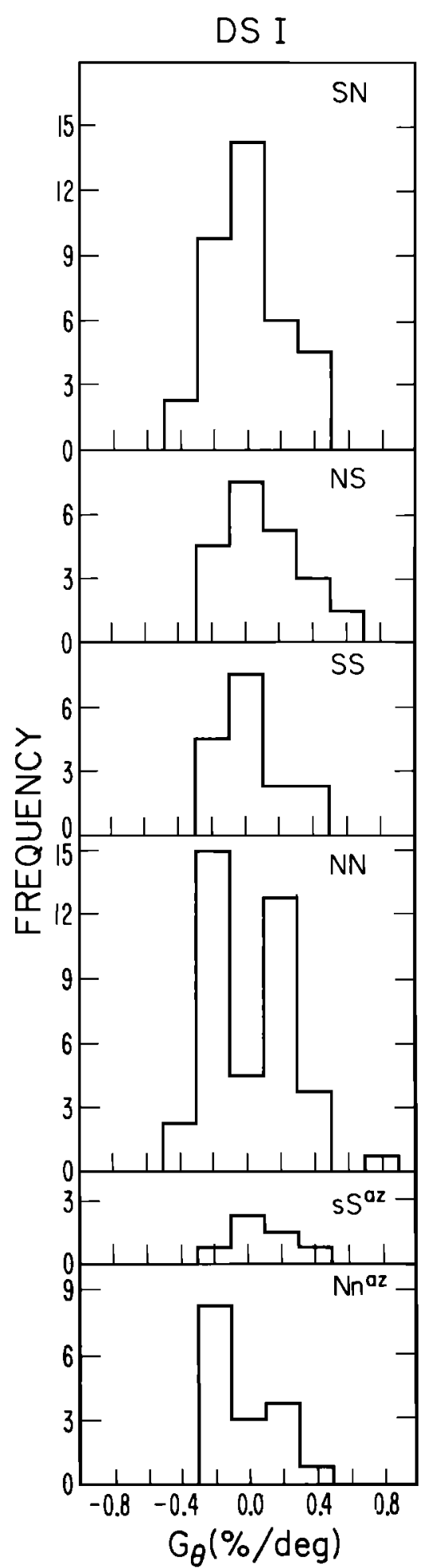

Fig. 7. $G_{\theta}$ for subsets of DS I. $\left\langle G_{\Lambda}\right\rangle^{a z}$ are all at least a factor of 3 larger than $\left\langle G_{\Lambda}^{*}\right\rangle^{a z}$ for $K_{0}=1.5 \times 10^{22} \mathrm{~cm}^{2} \mathrm{~s}^{-1}$ and are approximately equal to $\left\langle G_{\Lambda}{ }^{*}\right\rangle^{a z}$ for $K_{0}=5 \times 10^{21} \mathrm{~cm}^{2} \mathrm{~s}^{-1}$. The relative increase in $\left\langle G_{\Lambda}\right\rangle$ due to applying restrictions is larger in the experimental analysis than in the model analyses, although the large uncertainty in $\left\langle G_{\Lambda}\right\rangle$ allows the two increases to be consistent. Thus some differences exist between experimental and model results, but overall similarities in the two analyses are clearly evident.

The results for the latitudinal restriction are consistent with our initial interpretation that the sector lengths may be a measure of the amplitude of the current sheet waves. The latitude restriction selects a subset of the $N N$ data (see Figure 8) in which the Voyager 1 fluxes in longer sectors are generally lower than the Voyager 2 fluxes in shorter sectors, an effect which is consistent with Voyager 1 being more distant from a local flux intensity maximum at the current sheet than Voyager 2. The complementary subset of $N N$ data eliminated by the latitude restriction, i.e., those days with Voyager 2 sectors longer than Voyager 1 sectors, is consistent with Voyager 2 being further from the current sheet than Voyager 1.

Under restricted interplanetary conditions our measurements during the period from mid-1981 until mid-1983 are consistent with the interpretation that there exists a small, negative latitudinal gradient with respect to the heliospheric current sheet in the outer solar system as would result from the presence of local intensity minima symmetrically displaced from the current sheet in the two hemispheres. Further studies are required to determine whether such gradients are typical of other time periods.

Our results for the latitudinal gradient can be most directly compared with the gradient derived from single-point observations by experiments on IMP 8 at $1 \mathrm{AU}$ reported by Newkirk et al. [this issue] and summarized in Table 4. Although exact quantitative comparison is not straightforward because of the different measuring techniques, it does appear that there are no large differences in these "angular" gradients, even though measured in subsequent 11-year solar cycles and at different radial locations. Understanding the implications of this similarity for the relative importance of drifts and perpendicular diffusion requires further consideration of the dependence of model calculations on these factors.

\section{Discussion}

A number of features have not yet been addressed by theoretical modeling or by most of the experimental studies. Those features, which may have considerable and as yet unknown effects on our understanding of drift-dominated cosmic ray transport, are the effects of complicated current sheet topologies, variable solar wind speeds, and large-scale magnetic field intensity perturbations. For example, high-speed solar wind streams restructure the interplanetary medium by overtaking and interacting with slower speed solar wind as they propagate outward from the sun, generating corotating interaction regions (CIRs) [e.g., Smith and Wolfe, 1979] or merged

TABLE 4. Recent Estimates of Cosmic Ray Angular Gradient

\begin{tabular}{cccccc}
\hline Study & $\begin{array}{c}\text { AU } \\
\text { Time }\end{array}$ & $\begin{array}{c}\text { Energy } \\
\text { Period }\end{array}$ & $\begin{array}{c}\text { Threshold, } \\
\text { MeV }\end{array}$ & $\begin{array}{c}\text { Median } \\
\text { Rigidity, } \\
\text { GV }\end{array}$ & Gradient \\
\hline $\begin{array}{c}\text { Newkirk et al. } \\
\text { [this issue] }\end{array}$ & 1 & $1974-1977$ & 106 & 0.9 & $G_{\lambda_{m a}}=-0.4 \% / \operatorname{deg}(-23 \% / \mathrm{AU}$ at $1 \mathrm{AU})$ \\
\begin{tabular}{c} 
This study \\
\hline
\end{tabular} & $\sim 12$ & $1981-1983$ & 75 & 1.6 & $G_{\Lambda}=-0.2 \% / \mathrm{deg}(-1 \% / \mathrm{AU}$ at $12 \mathrm{AU})$ \\
\hline
\end{tabular}

Measurements of cosmic rays with kinetic energies greater than energy threshold. 


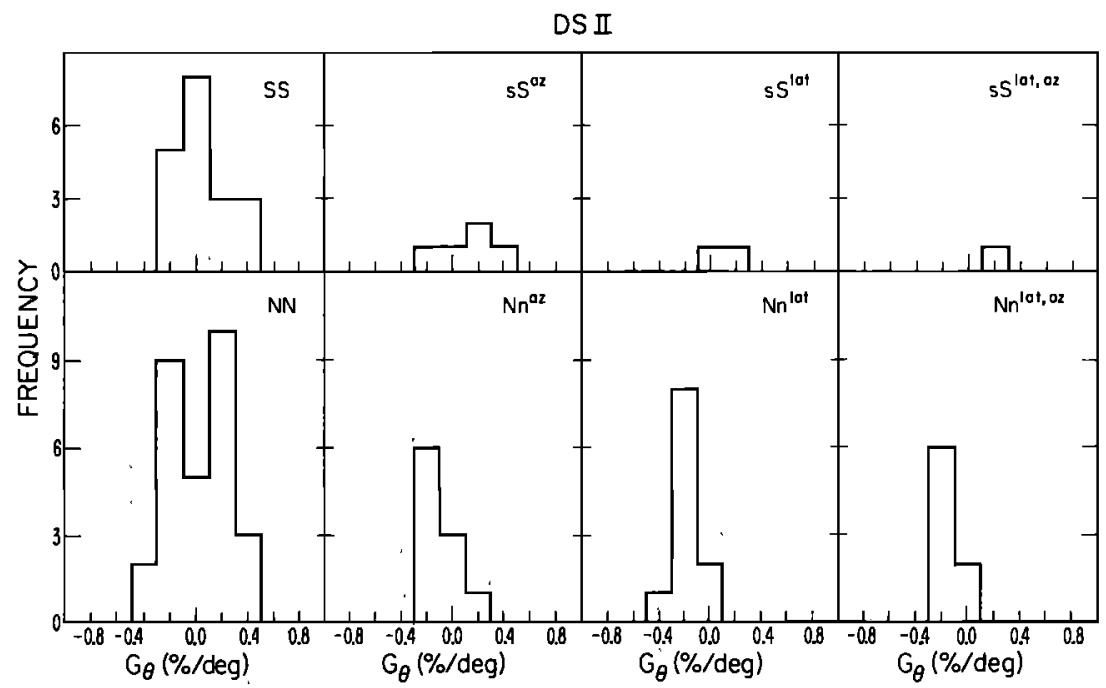

Fig. 8. $G_{\theta}$ for the unrestricted and restricted subsets of DS II discussed in the text. The various restricted subsets show a separation of means and modes, whereas the unrestricted subsets do not show a separation.

interaction regions [e.g., Burlaga et al., 1983; Burlaga, 1984]. The effect of the presence of CIRs on cosmic ray transport has been addressed in a number of studies [e.g., Morfill et al., 1980; Duggal et al:, 1981; Thomas and Gall, 1982; Newkirk and Fisk, 1985], with quite different interpretations. Most sector boundarles in the outer solar system are expected to be embedded in CIRs (see, for example, the discussion of Figures 7-9 by Thomas and Smith [1981]) or in merged interaction regions [e.g., Burlaga et al., 1985], so that their presence at each of our sector boundaries is implicit, and therefore we have not specifically addressed effects due to the interaction regions observed by the Voyager spacecraft during this period [Burlaga et al., 1985]. As Voyager 1 continues to rise in heliograhic latitude and as the sun approaches a new period of minimum activity, the effects of CIRs on cosmic rays should be monitored closely in order to detect any changes as the spacecraft encounters the heliospheric current sheet less frequently. In addition, CIRs both with and without embedded

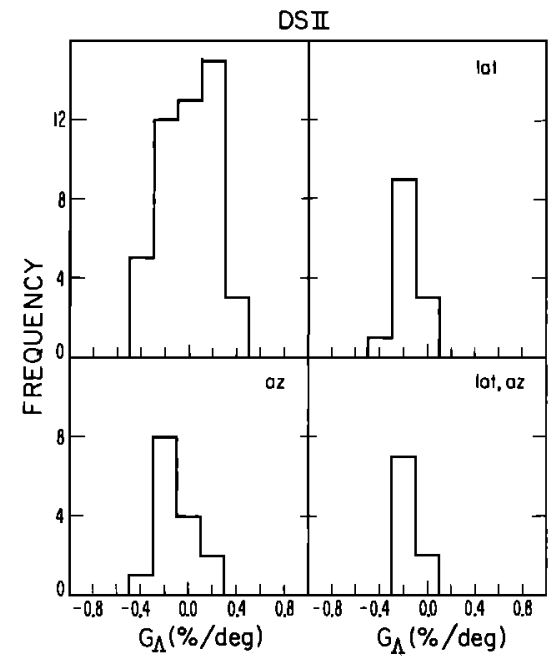

Fig. 9. Estimates of $G_{\Lambda}$, the latitude gradient, with respect to the current sheet for the unrestricted and restricted subsets of DS II discussed in the text. The various restricted subsets indicate a decrease in flux with increasing distance from the current sheet, whereas the unrestricted subset does not. current sheets should be incorporated into the threedimensional model calculations in order to further investigate the relative importance of CIRs and current sheets on measurements of the type presented in this paper.

\section{ConClusions}

We have combined measurements of the interplanetary magnetic field and fluxes of $>75-\mathrm{MeV}$ protons at the Voyager spacecraft in the outer solar system from mid-1981 to mid1983 to derive $\left\langle G_{\Lambda}\right\rangle$, an estimate of the latitudinal gradient of cosmic ray intensity with respect to the heliospheric current sheet. We find (1) results consistent with a zero gradient when the magnetic field is disturbed and magnetic field sectors are not well defined and (2) a statistically significant result $\left(\left\langle G_{\Lambda}\right\rangle=-0.22 \pm 0.03 \% / \mathrm{deg}\right)$ when the solar wind and particle fluxes are undisturbed by Forbush decreases and solar flares and when sectors are well defined or only moderately disturbed. The result for undisturbed times indicates a net decrease in cosmic ray intensity with increasing distance from the heliospheric current sheet in the outer solar system during the present portion of the 22-year solar magnetic activity cycle. Our result is qualitatively consistent with various aspects of predictions for $\sim 1-G V$ protons from a threedimensional model of heliospheric particle transport including drift, in which the current sheet organizes cosmic ray flux, and is in general agreement with the experimental results of Newkirk et al. [this issue] for cosmic rays at $1 \mathrm{AU}$. It is important both to improve the model calculations and to extend the measurements to solar minimum conditions, when the interplanetary medium should have the simplest structure.

Acknowledgments. We are grateful to R. E. Vogt for his contributions to the Cosmic Ray Subsystem on the Voyager spacecraft and to N. F. Ness, the principal investigator for the GSFC MAG experiment, for the magnetic field data from the Voyager spacecraft used in this study. We appreciate the contributions of the other Voyager Cosmic Ray subsystem team members - scientists and engineers at the California Institute of Technology, the Goddard Space Flight Center, the University of Arizona, and the University of New Hampshire. Work at Caltech was supported in part by the National Aeronautics and Space Administration under contract JPL 49-556-631200-2600 and grant NGR 05-002-160. Work at Arizona was supported in part by the National Aeronautics and Space Administration under grant NSG 7101, by the National Science Foundation grant ATM220-18, and by the International Study Program of the National Sci- 
ence Foundation. The idea for this study originated in discussions at a meeting of the Cosmic Ray Modulation Workshop.

The Editor thanks R. Decker and G. Newkirk for their assistance in evaluating this paper.

\section{REFERENCES}

Badruddin, R. S. Yadav, and N. R. Yadav, Intensity variation of cosmic rays near the heliospheric current sheet, Planet. Space Sci., 33, 191, 1985.

Behannon, K. W., M. H. Acuna, L. F. Burlaga, R. P. Lepping, N. F. Ness, and F. M. Neubauer, Magnetic field experiment for Voyagers 1 and 2, Space Sci. Rev., 21, 235, 1977.

Behannon, K. W., L. F. Burlaga, and A. J. Hundhausen, A comparison of coronal and interplanetary current sheet inclinations, J. Geophys. Res., 88, 7837, 1983.

Burlaga, L. F., MHD processes in the outer heliosphere, Space Sci. Rev., 39, 255, 1984.

Burlaga, L. F., R. Schwenn, and H. Rosenbauer, Dynamical evolution of interplanetary magnetic fields and flows between $0.3 \mathrm{AU}$ and 8.5 AU: Entrainment, Geophys. Res. Lett., 10, 413, 1983.

Burlaga, L. F., F. B. McDonald, M. L. Goldstein, and S. J. Lazarus, Cosmic ray modulation and turbulent interaction regions near 11 AU, J. Geophys. Res., 90, 12,027, 1985.

Christon, S. P., A. C. Cummings, E. C. Stone, K. W. Behannon, and L. F. Burlaga, Differential measurement of cosmic-ray gradient with respect to interplanetary current sheet, Proc. Int. Cosmic Ray Conf. 19th, 4, 445, 1985.

Cummings, A. C., and W. R. Webber, Temporal variations of the anomalous oxygen component, Solar Wind Five, NASA Conf. Publ., CP-2280, 427, 1983.

Decker, R. B., S. M. Krimigis, and D. Venkatesan, Estimate of cosmic-ray latitudinal gradient in 1981-1982, Astrophys. J. Lett., 278, L1 19, 1984.

Duggal, S. P., B. T. Tsurutani, M. A. Pomerantz, C. H. Tsao, and E. J. Smith, Relativistic cosmic rays and corotating interaction regions, J. Geophys. Res., 86, 7473, 1981.

Fisk, L. A., Solar modulation of cosmic rays, 4, Latitude-dependent modulation, J. Geophys. Res., 81, 4646, 1976.

Hoeksema, J. T., Structure and evolution of the large scale heliospheric magnetic fields, Ph.D. thesis, Stanford University, Stanford, Calif., 1984

Jokipii, J. R., and J. M. Davila, Effects of drift on the transport of cosmic rays, V, More realistic diffusion coefficients, Astrophys. J., 248, 1156, 1981.

Jokipii, J. R., and J. Kota, Cosmic rays near the heliospheric current sheet, 2, Ensemble approach to comparing theory and observations, J. Geophys. Res., this issue.

Jokipii, J. R., E. H. Levy, and W. B. Hubbard, Effects of drift on the transport of cosmic rays, I. General properties, application to solar modulation, Astrophys. J., 213, 861, 1977.

Klein, L., and L. F. Burlaga, Interplanetary sector boundaries 19711973, J. Geophys. Res., 85, 2269, 1980.

Kota, J., and J. R. Jokipii, Effects of drift on the transport of cosmic rays, VI, A three dimensional model including diffusion, Astrophys. $J ., 265,573,1983$.

Lockwood, J. A., and W. R. Webber, Integral radial cosmic ray gradi- ents in the solar system from 1972 to 1982 , Astrophys. J., 279, 151, 1984.

Morfill, G., M. Sholer, and M. A. I. Van Hollebeke, The longitudinal galactic cosmic ray intensity modulation in a diffusive and a scatter-free model of the inner heliosphere, J. Geophys. Res., 85, 2307, 1980.

Newkirk, G., and L. A. Fisk, Variation of cosmic rays and solar wind properties with respect to the heliospheric current sheet, 1, Five$\mathrm{GeV}$ protons and solar wind speed, J. Geophys. Res., 90, 3391, 1985.

Newkirk, G., and J. A. Lockwood, Cosmic ray gradients in the heliosphere and particle drifts, Geophys. Res. Lett., 8, 619, 1981.

Newkirk, G., J. Asbridge, J. A. Lockwood, M. Garcia-Munoz, and J. A. Simpson, Variation of cosmic rays and solar wind properties with respect to the heliospheric current sheet, 2, Rigidity dependence of the latitudinal gradient of cosmic rays at $1 \mathrm{AU}, J$. Geophys. Res., this issue.

Seuss, S. T. and J. Feynman, Sector boundary distortion in the interplanetary medium, J. Geophys. Res., 82, 2405, 1977.

Smith, E. J., and J. H. Wolfe, Fields and plasmas in the outer solar system, Space Sci. Rev., 23, 217, 1979.

Stone, E. C., R. E. Vogt, F. B. McDonald, B. J. Teegarden, J. H. Trainor, J. R. Jokipii, and W. R. Webber, Cosmic ray investigation for the Voyager missions; energetic particle studies in the outer heliosphere-and beyond, Space Sci. Rev., 21, 335, 1977.

Svalgaard, L., and J. M. Wilcox, Structure of the extended solar magnetic field and the sunspot cycle variation in cosmic ray intensity, Nature, 262, 766, 1976.

Thomas, B. T., and R. Gall, The effect of corotating interaction regions on the propagation of relativistic cosmic rays in the heliosphere, J. Geophys. Res., 87, 4542, 1982.

Thomas, B. T., and E. J. Smith, The structure and dynamics of the heliospheric current sheet, J. Geophys. Res., 86, 11,105, 1981.

Tritakis, V. P., Heliospheric current sheet displacements during the solar cycle evolution, J. Geophys. Res., 89, 6588, 1984.

Van Allen, J. A., and B. A. Randall, Interplanetary cosmic ray intensity: 1972-1984 and out to 32 AU, J. Geophys. Res., 90, 1399, 1985.

Venkatesan, D., R. B. Decker, and S. M. Krimigis, Radial gradient of cosmic ray intensity from a comparative study of data from Voyager 1 and 2 and IMP 8, J. Geophys. Res., 89, 3735, 1984.

Venkatesan, D., R. B. Decker, S. M. Krimigis, and J. A. Van Allen, The galactic cosmic ray intensity minimum in the inner and outer heliosphere in solar cycle 21, J. Geophys. Res., 90, 2905, 1985.

K. W. Behannon and L. F. Burlaga, Goddard Space Flight Center, Greenbelt, MD 20771.

S. P. Christon, A. C. Cummings, and E. C. Stone, California Institute of Technology, Pasadena, CA 91125.

J. R. Jokipii, University of Arizona, Tucson, AZ 85721.

J. Kota, Central Research Institute for Physics, Budapest, Hungary.

(Received July 31, 1985; revised October 1, 1985; accepted October 2,1985 .) 